\title{
Identification of Biomarkers Modulating Osteogenic Differentiation With Immune and Inflammation Landscapes in Mesenchymal Stem Cells: A Bioinformatics-based Comprehensive Study
}

\section{Ziyi Feng}

The First Affiliated Hospital of China Medical University

\section{Xin Su}

The First Affiliated Hospital of China Medical University

\section{Ting Wang}

The First Affiliated Hospital of China Medical University

Shu Guo ( $\sim$ sguo@cmu.edu.cn )

The First Affiliated Hospital of China Medical University

\section{Research Article}

Keywords: bioinformatics, osteogenesis, mesenchymal stem cells, biomarker, immune, inflammation

Posted Date: January 31st, 2022

DOI: https://doi.org/10.21203/rs.3.rs-1281574/v1

License: (c) (i) This work is licensed under a Creative Commons Attribution 4.0 International License. Read Full License 


\section{Abstract}

Background: Inducing osteogenic mesenchymal stem cell (MSC) differentiation brings a huge potential for clinical bone defect repair. This present study is aimed to identify novel key biomarkers for osteogenesis of MSCs, and analyze their possible regulation roles on immune and inflammation during this process.

Results: Seven datasets (GSE159137, GSE159138, GSE114117, GSE88865, GSE153829, GSE63754, GSE73087) were obtained from the gene expression omnibus database, and 200 differentially expressed genes (DEGs) were identified from the train datasets. Biomarkers were screened through Logical regression of the selection operator algorithm, whose expressions and diagnostic performances were verified in the test datasets. FKBP prolyl isomerase 5 (FKBP5), insulin like growth factor binding protein 2, prostaglandin E receptor 2, SAM and HD domain containing deoxynucleoside triphosphate triphosphohydrolase 1 (SAMHD1) and transmembrane 0-mannosyltransferase targeting cadherins 1 were established to be osteogenesis-related biomarkers. Finally, the DEGs of immune and inflammation in osteogenesis were detected, and their correlation with the hub biomarkers were analyzed. The five selected biomarkers are strongly associated with immune and inflammation related genes.

Conclusion: Taken together, our manuscript identified five biomarkers related to stem cells osteogenesis and discussed their potential perspectives in immunoregulation, inflammation and tissue engineering during the process.

\section{Background}

Though omnipresent challenges exist in face of the clinical and surgical repairing of large-volume skeletal abnormalities, recent stem cell-based therapy (SCBT) has shown great potential for the treatment of severe bone defects $(1,2)$. In biological conditions, healthy bone tissues serve limited capabilities in self-renewal and regeneration which is majorly controlled by the activities of osteoclasts and osteoblasts. Nevertheless, such properties can be significantly suppressed, even halted in case of severe skeletal trauma or defects, resulting in great setbacks when taken into clinical consideration. Mesenchymal stem cells (MSCs), also known as mesenchymal stromal cells, including adipose-derived stem cells (ASCs) and bone-marrow stromal cells (BMSCs), plays a major role in regenerative medicine due to their fundamental properties in cell-regeneration and differentiation. They have high osteoinductive and osteogenic potential, which is theoretically ideal for bone regenerations and shows a promising alternative(3). Therefore, the search for and identification of important biochemical molecules and genes that may initiate or control seed osteogenic differentiation is vital for the establishment of relevant defect treatment strategies.

Depending on specific microenvironment, MSCs which have various properties in regulating immune and inflammation play crucial biological roles in ways of direct immune cell contact and the production of inflammatory regulatory molecules, namely, the paracrine effects $(4,5)$. Though the effect of "sensor and 
switcher of the immune system", MSCs have the capabilities of both up- and down- regulating inflammatory processes, mainly by responding to danger indicators and releasing anti-inflammatory mediators through activated Toll-like receptors (TLRs) ligands, such as TLR9 TLR7 and TLR2, and expressing the immune suppressors PD-L1 as well as PD-L2 under the stimulation of interferon gamma (IFN- $\gamma$ ), respectively(6). MSCs also has various effects in coordinating the migration, proliferation, and activation of immune cells according to different stages of osteogenesis and different categories of immune cells (7). And consistent with changes in proinflammatory and anti-inflammatory cytokines presenting in the micro-environment, MSCs secrete cytokines including prostaglandin E2 (PGE2), transforming growth factor beta (TGF- $\beta$ ), histocompatibility locus antigen-G (HLA-G) that induce the formation of regulatory $T$ cells, suppress neutrophil migration and more importantly, serve the role in immune-inhibition by monocyte/macrophage regulation(8-10). Therefore, exploring the potential roles of immunomodulation and inflammatory regulation which involved MSC osteogenic differentiation is crucial in discovering novel therapeutic strategies and improving currently inefficient bone remodeling.

In this study, we performed a comprehensive strategy to determine biomarkers related to osteogenic differentiation of MSCs, and further investigated the relationship between those biomarkers and genes involving immunoregulation and inflammation participating in this process. The objective of our research is to form a basis for further explorations of significant genes, biochemical pathways as well as vital bioactive molecules affecting the osteogenic role of mesenchymal stem cells.

\section{Methods}

\section{Data Collection and Processing}

The study flowchart is presented in Figure 1. Three datasets which expression profiling by high throughput sequencing (GSE159137, GSE159138, GSE114117) and four datasets which expression profiling by array (GSE88865, GSE153829, GSE63754, GSE73087) were retrieved from the GEO database (http://www.ncbi.nlm.nih.gov/geo), and the characteristics of these included datasets were presented in Table 1. The three high throughput sequencing were selected as the train group containing a total of 7 uninduced MSCs samples and 12 osteogenic induced MSCs samples. And four datasets which expression profiling by array, with a total of 10 uninduced MSCs samples and 18 osteogenic MSCs induced samples, were utilized for test validation. The RNA algorithm was applied to background correction and data normalization(39). Then, probes were transmuted into their corresponding gene symbols based on platform annotation information.

\section{Identification of Differentially Expressed Genes}

The analysis of differentially expressed genes (DEGs) was performed by using the "limma" R package (http://www.bioconductor.org/packages/release/bioc/html/limma.html). Using false discovery rate (FDR) q-value $<0.05$ and log2 fold change $(F C) \geq 2$ as the filter condition. "Pheatmap" R package (https://cran.r-project.org/web/packages/pheatmap/index.html) , "ggplot2" 
(https://www.rdocumentation.org/packages/ggplot2/versions/2.1.0) R package and "ggrepel" R package (https://cloud.r-project.org/package=ggrepel) were utilized to visualize DEGs.

\section{Enrichment Analyses of DEGs}

Gene Ontology (GO) (http://geneontology.org/) and the Kyoto Encyclopedia of Genes and Genomes (KEGG) (https://www.kegg.jp/) were used for functional and pathway enrichment analyses, respectively. Disease Ontology (DO) (http://disease-ontology.org) comprises 8043 inherited, acquired, and developmental human diseases. Based on molecular signature database, Gene Set Enrichment Analysis (GSEA) (http://software.broadinstitute.org/gsea/index.jsp) is a statistical tool for interpreting gene expression data. GSEA 4.1.0 was utilized to predict the potential functions and downstream access of the two clusters. Prior to enrichment analyses, "org.Hs.eg.db" R package (http://www.bioconductor.org/packages/release/data/annotation/html/org.Hs.eg.db.html), a human genome annotation database was used for conversion of gene symbol codes into Entrez ID. FDR q-value $<0.05$ were considered markedly enriched for GO terms, DO and GSEA analyses, while p-value $<0.05$ was considered significant for KEGG analyses.

\section{Screening for MSCs osteogenesis related biomarkers}

LASSO was used to investigate osteogenesis-related biomarkers via R package "glmnet" R package (https://mirrors.sjtug.sjtu.edu.cn/cran/web/packages/glmnetSE/index.html) in train group. while "pROC" R package (https://cran.rstudio.com/web/packages/pROC/index.html) was used for computing the area under the curve (AUC) in train and test group. Accuracy, sensitivity, and specificity of a receiver operating characteristic (ROC) curve to assess the diagnostic ability of the markers. $p<0.05$ was the cut-off for significance.

\section{Selection of immune and inflammation related genes}

To make the research more comprehensive, we selected immune and inflammation related genes, and the differential expressed genes in osteogenesis and non-osteogenesis groups was visualized by using the methods mentioned above. PPI networks were established to assess the associations among genes in selected modules using the Search Tool for the Retrieval of Interacting Genes version 11 (STRING V11, https://string-preview.org/) with $>0.4$ as the confidence level. Cytoscape version 3.8.2 was used for network visualization. Hub genes, which are presented by highly interconnected nodes, may play vital roles in the PPI network.

\section{Interaction analysis of selected biomarkers}

Co-expression analysis of selected biomarkers with immune-related genes and inflammation-related genes was performed using "limma" in $\mathrm{R}$ (http://www.bioconductor.org/packages/release/bioc/html/limma.html), respectively. We then performed co-expression analysis, and the following parameters were used as filter conditions to select related genes: "correlation coefficient $=0.4$ " and "pvalueFilter $=0.05$ ". 


\section{Results}

\section{Identification of DEGs}

Differential expression analysis was conducted based on the train datasets to screen for DEGs. There were clear differences between osteogenesis MSCs and the control ones. From our heatmap (Figure 2A) and volcano plots (Figure 2B), we observed there were 200 DEGs. Among them, 156 genes were upregulated while 44 genes were downregulated.

\section{Functional enrichment analyses of DEGs}

With regards to biological processes, GO analysis showed that robust DEGs were mainly enriched in cellular divalent inorganic cation homeostasis, epithelial cell proliferation, and cellular calcium ion homeostasis in the biological process (BP) part. The cellular component (CC) part, the DEGs mainly mainly concentrated in collagen-containing extracellular matrix, postsynaptic density, and asymmetric synapse. In Molecular functions included peptide binding and amide binding among other important functions (Figure 3A). The KEGG analysis demonstrated that neuroactive ligand-receptor interaction, complement and coagulation cascades, as well as cytokine-cytokine receptor interaction were the most significant processes in MSCs osteogenic differentiation process (Figure 3B). DO analysis showed that DEGs were enriched in kidney disease and urinary system disease (Figure 4A).

Moreover, the GSEA analysis result indicated active pathways that varied between uninduced and osteogenic induced groups (Figure 4B), including cellular ion homeostasis, divalent inorganic cation homeostasis, inflammatory response, and metal ion homeostasis were established to be active in the osteogenic induced group. While the active pathways of uninduced group (Figure 4C) were chromosome segregation, polymeric cytoskeletal fiber, postsynapse, postsynaptic membrane, and synaptic membrane.

\section{Verification of the hub biomarkers}

Through LASSO logistic regression, a total of 7 genes were identified from robust DEGs as potential vitiligo-associated markers (Figure 5A). These 7 biomarkers included adrenoceptor alpha 1B (ADRA1B), FKBP prolyl isomerase 5 (FKBP5), insulin like growth factor binding protein 2 (IGFBP2), monoamine oxidase $A$ (MAOA), prostaglandin E receptor 2 (PTGER2), SAM and HD domain containing deoxynucleoside triphosphate triphosphohydrolase 1 (SAMHD1) and transmembrane 0mannosyltransferase targeting cadherins 1 (TMTC1).

To assess the accuracy for the biomarkers, we used 4 datasets (GSE88865, GSE153829, GSE63754, GSE73087) as verification sets to validate the efficacies of candidate biomarkers. Differential expressions of these five genes, including FKBP5, IGFBP2, PTGER2, SAMHD1 and TMTC1, were verified in test group, and their expressions were all significantly higher in the osteogenic induced groups (Figure 5B$\mathrm{H})$. The five biomarkers were all with an AUC more than 0.7 in the test datasets, and SAMHD1 presented the highest AUC of 0.861 , indicating the capabilities of the five biomarkers to diagnose MSCs osteogenic differentiation with excellent specificity and sensitivity (Figure 6). 


\section{Identification of DEGs related to immune and inflammation}

Heatmaps revealed 38 differentially expressing immune-related regulators between osteogenic induced and control groups with FDR q-value $<0.05$ (Figure 7A), with 31 upregulated and 7 downregulated. The interrelation of immune-related regulators was constructed using the STRING database, the proteinprotein interaction (PPI) network analysis (Figure 7B) indicated that IL6, LEP and ANGPT1 had the most interactions of the studied genes. GO and KEGG analyses were performed to the potential functions and pathways of the differentially expressing immune-related genes (Figure 8). The GO results revealed that the differentially expressing immune-related regulators were majorly concentrated in epithelial cell proliferation in BP, basolateral plasma membrane, receptor ligand activity in CC, receptor ligand activity and signaling receptor activator activity in MF. In KEGG, it concentrated in neuroactive ligand-receptor interaction, as well as cytokine-cytokine receptor interaction.

We screened differentially expressing inflammation-related regulators between osteogenic induced and control groups through $p$-value $<0.05$ and 32 upregulated and 18 downregulated genes were presented in Heatmaps (Figure 9A). The interaction as well as the expression number of inflammation-related regulators were shown in Figures 9B, CXCL8 and IL6 were treated as the central gene. GO and KEGG enrichment analyses found that response to molecule of bacterial origin and response to lipopolysaccharides in BP, secretory granule membrane and plasma membrane signaling receptor complex in CC, receptor ligand activity and signaling receptor activator activity in MF, while cytokinecytokine receptor interaction were the most important functions and pathways of the differentially expressing inflammation-related regulators according to the enrichment scores in KEGG (Figure 10).

\section{Potential roles of hub biomarkers in immune and inflammation}

We analyzed RNA-seq transcriptome to explore the association between differentially expressed immunerelated genes and selected biomarkers (Figure $11 \mathrm{~A}$ ) as well as the relationship between differentially expressed inflammation-related genes and the five hub biomarkers (Figure 11B). There seemed to be a regulating network of FKBP5, IGFBP2, PTGER2, SAMHD1, and TMTC1 with immune-related and inflammation-related genes. The correlation coefficients and positive and negative regulatory relationships between the genes we predicted are shown in the Table 2 and Table 3 . In the process of stem cell osteogenesis, the biomarkers we screened showed a strong correlation with immunity and inflammation, which laid a foundation for us to further study the mechanism of stem cell osteogenesis.

\section{Discussion}

Although the significance of MSCs in bone tissue engineering have been evaluated, their efficacy in osteogenic differentiation is limited without extra interventions in vivo and in vitro(11, 12). How to improve the efficiency of regenerative abilities of MSCs in damage restoration is still urgent. The search of novel key biomarkers in stem cell osteogenesis will be helpful in clarifying osteogenic potentials and mechanisms of stem cells, thus designing more appropriate bone filling materials to induce bone regeneration with increased proficiency and biocompatibility. 
In this study, we identified hub biomarkers of stem cells osteogenesis with a comprehensive strategy of LASSO logistic regression and ROC curve analysis. The five hub biomarkers we identified are FKBP5, IGFBP2, PTGER2, SAMHD1 and TMTC1. Although there have been studies focusing on exploring stemcell-related osteogenic biomarkers via bioinformatics analyses $(13,14)$, the hub markers SAMHD1 and FKBP5 we determined in this study have an average AUC over 0.8 in test datasets, indicating their reliable predictive abilities.

Among these 5 hub biomarkers, the effects of IGFBP2 and PTGER2 on osteogenesis have been reported, but remain controversial. IGFBP2, an important member of the insulin-like growth factor family, plays crucial roles in growth, development, and metabolism of human body $(15,16)$. Hamidouchel et al. demonstrated that IGFBP2 has the property of promoting the osteogenic differentiation of MSCs by enhancing the osteoblast phenotype genes, including RUNX2, ALP and COI1A1B(17). And consistent with our results, the expression of IGFBP2 increased significantly when inducing osteogenesis(18). Moreover, a recent study revealed that IGFBP2 is also capable of activating the JNK/Akt pathway and inducing the adipogenic differentiation of MSCs(19). Further researches are needed to elucidate the mechanisms and specific roles played by IGFBP2 in promoting osteogenesis or adipogenesis in MSCs. PTGER2 acts as a main receptor of prostaglandin E2, which has been shown to encourage osteogenic differentiation of MSCs, and stimulate the resorption and formation of bones(20-22). It is suggested in several current studies that local application of PTGER2 agonists to fractured rats in vitro can effectively stimulate the formation of bone marrow and periosteum, thus promoting fracture repairing(23-25). However, the direct effect of PTGER2 on the osteogenic differentiation of MSCs needs further research to confirm.

TMTC1, a glycosyltransferase, promotes 0-mannosylation of cadherin and maintains intracellular calcium homeostasis(26-28). Glycosylation is a key posttranslational modification process that participates in controlling extracellular matrix formation during osteogenesis $(29,30)$. GO analysis revealed that DEGs were mainly concentrated in extracellular matrix organization, and collagencontaining extracellular matrix. Previous studies have revealed that extracellular matrix modulates osteogenic effects by adhering to cytoskeletal proteins in MSCs(31, 32). Together with our results, TMTC1 and glycosylation may play crucial roles in the extracellular matrix formation during osteogenic differentiation.

The strong relationship between stem cells osteogenesis and immunity has been well reported within the osteoimmunology scope, and a controlled inflammatory response is vital for favoring osteogenesis(3335). In this study, we identified differentially expressing immune and inflammatory related regulators between osteogenic induced and uninduced groups, and numerous DEGs between groups were found, demonstrating a significant difference of immune and inflammation modulation between osteogenic and nonosteogenic status. And we further analyzed their association with hub biomarkers. Among the five biomarkers, SAMHD1 and FKBP5 represented to be most correlated with immune-related and inflammation-related genes in osteogenic differentiation. SAMHD1 is a deoxynucleoside triphosphohydrolase that can suppress innate immune responses to viral infection through interacting with various key proteins in immune signaling pathways $(36,37)$. FKBP5 is a stress-responsive molecule 
which also possesses the property of modulating immune function. Earlier researches have demonstrated the effects of SAMHD1 and FKBP5 on immune response and inflammation, but whether these effects present during the process of osteogenesis have not been fully elucidated(38). Our present results suggest that the potential mechanisms of SAMHD1 and FKBP5 exerting in osteogenesis might be greatly linked to immune and inflammation regulation.

In summary, we combined bioinformatic analysis and ROC analysis to screen the biomarkers associated with osteogenesis of stem cells, and analyzed their potential roles with immune and inflammation during this process. Although these hub genes are highly associated with osteogenesis of stem cells based on this study and other previous studies mentioned above, their biological mechanisms in osteogenesis remain unclear. Therefore, due further researches can be focused on specifying the precise mechanisms as well as discovering novel, related signaling pathways and bioactive molecules.

\section{Conclusion}

In conclusion, by combining bioinformatic and analysis, the current study has screened several hub biomarkers which are related to the osteogenic differentiation of mesenchymal stem cells, and analyzed their potential roles in immunomodulation and inflammatory responses during this process. It is suggested, by our results, the possible properties of these markers act through signaling pathways that are related to their effects in immune and inflammation regulation, also lay a foundation for us to further study the mechanism of mesenchymal stem cell osteogenesis.

\section{Declarations}

\section{Ethics approval and consent to participate}

Not applicable

\section{Consent for publication}

All authors have provided their consent for publication.

\section{Availability of data and materials}

The datasets generated and/or analyzed included in the manuscript are available in the GEO repository, http://www.ncbi.nlm.nih.gov/geo.

\section{Competing interests}

Not applicable

\section{Funding}

Not applicable 
Authors' contributions

SG and ZF conceived and designed the study. ZF and XS performed data acquisition and interpretation. ZF and TW wrote the paper. All authors approved the final manuscript.

\section{Acknowledgements}

We would like to thank all the anonymous colleagues who have made contributions to this manuscript.

\section{References}

1. Houschyar KS, Tapking C, Borrelli MR, Popp D, Duscher D, Maan ZN, et al. Wnt Pathway in Bone Repair and Regeneration - What Do We Know So Far. Front Cell Dev Biol. 2018;6:170.

2. Kangari P, Talaei-Khozani T, Razeghian-Jahromi I, Razmkhah M. Mesenchymal stem cells: amazing remedies for bone and cartilage defects. Stem Cell Res Ther. 2020;11(1):492.

3. Sui BD, Hu CH, Liu AQ, Zheng CX, Xuan K, Jin Y. Stem cell-based bone regeneration in diseased microenvironments: Challenges and solutions. Biomaterials. 2019;196:18-30.

4. Shi Y, Wang Y, Li Q, Liu K, Hou J, Shao C, et al. Immunoregulatory mechanisms of mesenchymal stem and stromal cells in inflammatory diseases. Nat Rev Nephrol. 2018;14(8):493-507.

5. Bernardo ME, Fibbe WE. Mesenchymal stromal cells: sensors and switchers of inflammation. Cell Stem Cell. 2013;13(4):392-402.

6. Jiang W, Xu J. Immune modulation by mesenchymal stem cells. Cell Prolif. 2020;53(1):e12712.

7. Qi K, Li N, Zhang Z, Melino G. Tissue regeneration: The crosstalk between mesenchymal stem cells and immune response. Cell Immunol. 2018;326:86-93.

8. Court AC, Le-Gatt A, Luz-Crawford P, Parra E, Aliaga-Tobar V, Bátiz LF, et al. Mitochondrial transfer from MSCs to T cells induces Treg differentiation and restricts inflammatory response. EMBO Rep. 2020;21(2):e48052.

9. Luque-Campos N, Contreras-López RA, Jose Paredes-Martínez M, Torres MJ, Bahraoui S, Wei M, et al. Mesenchymal Stem Cells Improve Rheumatoid Arthritis Progression by Controlling Memory T Cell Response. Front Immunol. 2019;10:798.

10. Joel MDM, Yuan J, Wang J, Yan Y, Qian H, Zhang X, et al. MSC: immunoregulatory effects, roles on neutrophils and evolving clinical potentials. Am J Transl Res. 2019;11(6):3890-904.

11. Chen Q, Shou P, Zheng C, Jiang M, Cao G, Yang Q, et al. Fate decision of mesenchymal stem cells: adipocytes or osteoblasts? Cell Death Differ. 2016;23(7):1128-39.

12. Gibon E, Lu L, Goodman SB. Aging, inflammation, stem cells, and bone healing. Stem Cell Res Ther. 2016;7:44.

13. Khodabandehloo F, Taleahmad S, Aflatoonian R, Rajaei F, Zandieh Z, Nassiri-AsI M, et al. Microarray analysis identification of key pathways and interaction network of differential gene expressions during osteogenic differentiation. Hum Genomics. 2020;14(1):43. 
14. Fan T, Qu R, Yu Q, Sun B, Jiang X, Yang Y, et al. Bioinformatics analysis of the biological changes involved in the osteogenic differentiation of human mesenchymal stem cells. $\mathrm{J}$ Cell Mol Med. 2020;24(14):7968-78.

15. Haywood NJ, Slater TA, Matthews CJ, Wheatcroft SB. The insulin like growth factor and binding protein family: Novel therapeutic targets in obesity \& diabetes. Mol Metab. 2019;19:86-96.

16. Khan S, Lu X, Huang Q, Tang J, Weng J, Yang Z, et al. IGFBP2 Plays an Essential Role in Cognitive Development during Early Life. Adv Sci (Weinh). 2019;6(23):1901152.

17. Hamidouche Z, Fromigué 0 , Ringe J, Häupl T, Marie PJ. Crosstalks between integrin alpha 5 and IGF2/IGFBP2 signalling trigger human bone marrow-derived mesenchymal stromal osteogenic differentiation. BMC Cell Biol. 2010;11:44.

18. Kanzaki S, Baxter RC, Knutsen R, Baylink DJ, Mohan S. Evidence that human bone cells in culture secrete insulin-like growth factor (IGF)-II and IGF binding protein-3 but not acid-labile subunit both under basal and regulated conditions. J Bone Miner Res. 1995;10(6):854-8.

19. Wang Y, Liu Y, Fan Z, Liu D, Wang F, Zhou Y. IGFBP2 enhances adipogenic differentiation potentials of mesenchymal stem cells from Wharton's jelly of the umbilical cord via JNK and Akt signaling pathways. PLoS One. 2017;12(8):e0184182.

20. Ern C, Frasheri I, Berger T, Kirchner HG, Heym R, Hickel R, et al. Effects of prostaglandin E(2) and D(2) on cell proliferation and osteogenic capacity of human mesenchymal stem cells. Prostaglandins Leukot Essent Fatty Acids. 2019;151:1-7.

21. Feigenson M, Eliseev RA, Jonason JH, Mills BN, O'Keefe RJ. PGE2 Receptor Subtype 1 (EP1) Regulates Mesenchymal Stromal Cell Osteogenic Differentiation by Modulating Cellular Energy Metabolism. J Cell Biochem. 2017;118(12):4383-93.

22. Liu Y, Yao Q, Sun H. Prostaglandin E2 Modulates Bone Morphogenetic Protein-2 Induced Osteogenic Differentiation on a Biomimetic 3D Nanofibrous Scaffold. J Biomed Nanotechnol. 2018;14(4):74755 .

23. Li M, Thompson DD, Paralkar VM. Prostaglandin E(2) receptors in bone formation. Int Orthop. 2007;31(6):767-72.

24. Li M, Ke HZ, Qi H, Healy DR, Li Y, Crawford DT, et al. A novel, non-prostanoid EP2 receptor-selective prostaglandin E2 agonist stimulates local bone formation and enhances fracture healing. J Bone Miner Res. 2003;18(11):2033-42.

25. Paralkar VM, Borovecki F, Ke HZ, Cameron KO, Lefker B, Grasser WA, et al. An EP2 receptor-selective prostaglandin E2 agonist induces bone healing. Proc Natl Acad Sci U S A. 2003;100(11):6736-40.

26. Larsen ISB, Narimatsu Y, Joshi HJ, Siukstaite L, Harrison OJ, Brasch J, et al. Discovery of an Omannosylation pathway selectively serving cadherins and protocadherins. Proc Natl Acad Sci U S A. 2017;114(42):11163-8.

27. Eisenhaber B, Sinha S, Jadalanki CK, Shitov VA, Tan QW, Sirota FL, et al. Conserved sequence motifs in human TMTC1, TMTC2, TMTC3, and TMTC4, new 0-mannosyltransferases from the GT-C/PMT clan, are rationalized as ligand binding sites. Biol Direct. 2021;16(1):4. 
28. Sunryd JC, Cheon B, Graham JB, Giorda KM, Fissore RA, Hebert DN. TMTC1 and TMTC2 are novel endoplasmic reticulum tetratricopeptide repeat-containing adapter proteins involved in calcium homeostasis. J Biol Chem. 2014;289(23):16085-99.

29. Sun Y, Weng Y, Zhang C, Liu Y, Kang C, Liu Z, et al. Glycosylation of Dentin Matrix Protein 1 is critical for osteogenesis. Sci Rep. 2015;5:17518.

30. Li C, Armstrong JP, Pence IJ, Kit-Anan W, Puetzer JL, Correia Carreira S, et al. Glycosylated superparamagnetic nanoparticle gradients for osteochondral tissue engineering. Biomaterials. 2018;176:24-33.

31. Assis-Ribas T, Forni MF, Winnischofer SMB, Sogayar MC, Trombetta-Lima M. Extracellular matrix dynamics during mesenchymal stem cells differentiation. Dev Biol. 2018;437(2):63-74.

32. Stanton AE, Tong X, Yang F. Extracellular matrix type modulates mechanotransduction of stem cells. Acta Biomater. 2019;96:310-20.

33. Medhat D, Rodríguez Cl, Infante A. Immunomodulatory Effects of MSCs in Bone Healing. Int J Mol Sci. 2019;20(21).

34. Qiu P, Li M, Chen K, Fang B, Chen P, Tang Z, et al. Periosteal matrix-derived hydrogel promotes bone repair through an early immune regulation coupled with enhanced angio- and osteogenesis. Biomaterials. 2020;227:119552.

35. Kovach TK, Dighe AS, Lobo PI, Cui Q. Interactions between MSCs and immune cells: implications for bone healing. J Immunol Res. 2015;2015:752510.

36. Chen S, Bonifati S, Qin Z, St Gelais C, Wu L. SAMHD1 Suppression of Antiviral Immune Responses. Trends Microbiol. 2019;27(3):254-67.

37. Chen S, Bonifati S, Qin Z, St Gelais C, Kodigepalli KM, Barrett BS, et al. SAMHD1 suppresses innate immune responses to viral infections and inflammatory stimuli by inhibiting the NF-KB and interferon pathways. Proc Natl Acad Sci U S A. 2018;115(16):E3798-e807.

38. Zannas AS, Jia M, Hafner K, Baumert J, Wiechmann T, Pape JC, et al. Epigenetic upregulation of FKBP5 by aging and stress contributes to NF-KB-driven inflammation and cardiovascular risk. Proc Natl Acad Sci U S A. 2019;116(23):11370-9.

39. McCall MN, Bolstad BM, Irizarry RA. Frozen robust multiarray analysis (fRMA). Biostatistics. 2010;11(2):242-53.

\section{Tables}

Table 1. Gene expression data from Gene Expression Omnibus (GEO) database. 


\begin{tabular}{|lllllll|}
\hline Dataset ID & Total samples & Control & Treat & Data type & Tissue type & Country \\
\hline GSE159137 & 6 & 6 & 0 & RNA-seq & AMSCs & China \\
\hline GSE159138 & 6 & 0 & 6 & RNA-seq & AMSCs & China \\
\hline GSE114117 & 7 & 1 & 6 & RNA-seq & hMSCs & China \\
\hline GSE88865 & 6 & 3 & 3 & Microarray & PDMSCs & China \\
\hline GSE153829 & 6 & 0 & 6 & Microarray & MSCs & China \\
\hline GSE63754 & 6 & 3 & 3 & Microarray & ADSCs & Lithuania \\
\hline GSE73087 & 7 & 1 & 6 & Microarray & MSCs & China \\
\hline
\end{tabular}

Table 2. Co-expression of biomarkers and immune related genes. 


\begin{tabular}{|c|c|c|c|c|}
\hline Immune related gene & Biomarker & cor & $p$ value & Regulation \\
\hline SAMHD1 & FKBP5 & 0.878497 & 0.000169 & positive \\
\hline APOD & FKBP5 & 0.841998 & 0.000591 & positive \\
\hline SAA1 & FKBP5 & 0.657604 & 0.020119 & positive \\
\hline TSC22D3 & FKBP5 & 0.632426 & 0.027332 & positive \\
\hline ANGPT1 & FKBP5 & 0.698622 & 0.011488 & positive \\
\hline PTK2B & FKBP5 & 0.784954 & 0.002492 & positive \\
\hline ANOS1 & FKBP5 & -0.71911 & 0.008395 & negative \\
\hline NRG1 & FKBP5 & -0.61721 & 0.032506 & negative \\
\hline AGTR1 & FKBP5 & 0.791893 & 0.002142 & positive \\
\hline EDNRB & FKBP5 & 0.612917 & 0.034084 & positive \\
\hline PDGFD & FKBP5 & 0.728478 & 0.00721 & positive \\
\hline SDC1 & FKBP5 & -0.60023 & 0.039067 & negative \\
\hline C7 & FKBP5 & 0.679423 & 0.015088 & positive \\
\hline C5AR2 & FKBP5 & 0.645377 & 0.023422 & positive \\
\hline GDF7 & FKBP5 & 0.65612 & 0.0205 & positive \\
\hline EPGN & FKBP5 & -0.65542 & 0.020681 & negative \\
\hline A2M & FKBP5 & 0.645669 & 0.023339 & positive \\
\hline UCN2 & FKBP5 & 0.678846 & 0.015208 & positive \\
\hline PTH1R & FKBP5 & 0.577018 & 0.049494 & positive \\
\hline NGFR & FKBP5 & 0.746981 & 0.005242 & positive \\
\hline SAMHD1 & IGFBP2 & 0.638202 & 0.025534 & positive \\
\hline APOD & IGFBP2 & 0.812225 & 0.001329 & positive \\
\hline LGR4 & IGFBP2 & -0.77284 & 0.003206 & negative \\
\hline ANGPTL4 & IGFBP2 & 0.733831 & 0.006592 & positive \\
\hline PTK2B & IGFBP2 & 0.645715 & 0.023325 & positive \\
\hline NRG1 & IGFBP2 & -0.65704 & 0.020262 & negative \\
\hline AGTR1 & IGFBP2 & 0.781886 & 0.00266 & positive \\
\hline PDGFD & IGFBP2 & 0.611956 & 0.034444 & positive \\
\hline
\end{tabular}




\begin{tabular}{|c|c|c|c|c|}
\hline LEP & IGFBP2 & 0.740169 & 0.005912 & positive \\
\hline SDC1 & IGFBP2 & -0.77122 & 0.003313 & negative \\
\hline NR2F1 & IGFBP2 & 0.718353 & 0.008497 & positive \\
\hline C7 & IGFBP2 & 0.814873 & 0.001244 & positive \\
\hline EPGN & IGFBP2 & -0.87914 & 0.000165 & negative \\
\hline STC1 & IGFBP2 & 0.635467 & 0.026375 & positive \\
\hline A2M & IGFBP2 & 0.703206 & 0.010733 & positive \\
\hline CCL13 & IGFBP2 & 0.701208 & 0.011058 & positive \\
\hline UCN2 & IGFBP2 & 0.816073 & 0.001206 & positive \\
\hline PTH1R & IGFBP2 & 0.645509 & 0.023384 & positive \\
\hline NGFR & IGFBP2 & 0.703752 & 0.010645 & positive \\
\hline SAMHD1 & SAMHD1 & 1 & 1.03E-70 & positive \\
\hline APOD & SAMHD1 & 0.813539 & 0.001286 & positive \\
\hline PTGER2 & SAMHD1 & 0.586136 & 0.04519 & positive \\
\hline SAA1 & SAMHD1 & 0.698088 & 0.011579 & positive \\
\hline MMP7 & SAMHD1 & 0.597863 & 0.040052 & positive \\
\hline TSC22D3 & SAMHD1 & 0.652469 & 0.021461 & positive \\
\hline ANGPT1 & SAMHD1 & 0.626446 & 0.029289 & positive \\
\hline PTK2B & SAMHD1 & 0.653912 & 0.021078 & positive \\
\hline ANOS1 & SAMHD1 & -0.7379 & 0.006149 & negative \\
\hline AGTR1 & SAMHD1 & 0.706966 & 0.010141 & positive \\
\hline GDF7 & SAMHD1 & 0.661101 & 0.01924 & positive \\
\hline EPGN & SAMHD1 & -0.65035 & 0.022035 & negative \\
\hline SLC40A1 & SAMHD1 & 0.679409 & 0.015091 & positive \\
\hline A2M & SAMHD1 & 0.616625 & 0.032717 & positive \\
\hline NGFR & SAMHD1 & 0.686714 & 0.013635 & positive \\
\hline IL6 & SAMHD1 & -0.57936 & 0.04836 & negative \\
\hline SAMHD1 & TMTC1 & 0.82189 & 0.001038 & positive \\
\hline PTGER2 & TMTC1 & 0.822676 & 0.001017 & positive \\
\hline
\end{tabular}




\begin{tabular}{|lllll|} 
LGR4 & TMTC1 & -0.65592 & 0.020552 & negative \\
\hline ANGPT1 & TMTC1 & 0.700543 & 0.011167 & positive \\
\hline ANOS1 & TMTC1 & 0.582698 & 0.046781 & positive \\
\hline NRG1 & TMTC1 & -0.79171 & 0.002151 & negative \\
\hline EDNRB & TMTC1 & -0.63645 & 0.026069 & negative \\
\hline C5AR1 & TMTC1 & 0.617272 & 0.032483 & positive \\
\hline C5AR2 & TMTC1 & 0.71421 & 0.00907 & positive \\
\hline IL1RL1 & TMTC1 & 0.639859 & 0.025034 & positive \\
\hline GDF7 & TMTC1 & 0.650512 & 0.02199 & positive \\
\hline SLC40A1 & TMTC1 & 0.840359 & 0.000621 & positive \\
\hline PPARGC1A & TMTC1 & 0.687872 & 0.013415 & positive \\
\hline AREG & TMTC1 & 0.665063 & 0.018279 & positive \\
\hline CRABP2 & TMTC1 & 0.6712 & 0.01686 & positive \\
\hline SAMHD1 & TMTC1 & -0.59813 & 0.039942 & negative \\
\hline PTGER2 & PTGER2 & 0.586136 & 0.04519 & positive \\
\hline ANOS1 & PTGER2 & 1 & $7.94 E-69$ & positive \\
\hline SLC40A1 & PTGER2 & -0.70111 & 0.011074 & negative \\
\hline PPARGC1A & PTGER2 & 0.630415 & 0.027979 & positive \\
\hline CRABP2 & PTGER2 & 0.80576 & 0.001556 & positive \\
\hline IL6 & PTGER2 & -0.80215 & 0.001694 & negative \\
\hline
\end{tabular}

Table 3. Co-expression of biomarkers and inflammation related genes. 


\begin{tabular}{|c|c|c|c|c|}
\hline Inflammation related gene & Biomarker & cor & $p$ value & Regulation \\
\hline BDKRB1 & FKBP5 & -0.65123958 & 0.021792337 & negative \\
\hline CD82 & FKBP5 & 0.863575418 & 0.00029466 & positive \\
\hline CDKN1A & FKBP5 & -0.782368639 & 0.002633147 & negative \\
\hline CHST2 & FKBP5 & 0.659470315 & 0.019646225 & positive \\
\hline CXCR6 & FKBP5 & 0.673761791 & 0.016292707 & positive \\
\hline INHBA & FKBP5 & -0.721062794 & 0.00813693 & negative \\
\hline LPAR1 & FKBP5 & 0.649708006 & 0.022209777 & positive \\
\hline MXD1 & FKBP5 & -0.680800725 & 0.014805463 & negative \\
\hline PTGER4 & FKBP5 & 0.70652552 & 0.010209257 & positive \\
\hline RIPK2 & FKBP5 & -0.632218742 & 0.027398517 & negative \\
\hline SLC4A4 & FKBP5 & -0.623010993 & 0.030456877 & negative \\
\hline TNFSF9 & FKBP5 & -0.58604887 & 0.045229839 & negative \\
\hline ADM & IGFBP2 & 0.619614804 & 0.03164453 & positive \\
\hline CD40 & IGFBP2 & 0.637875106 & 0.025633574 & positive \\
\hline CD82 & IGFBP2 & 0.905790243 & 0.0000498 & positive \\
\hline CDKN1A & IGFBP2 & -0.646818915 & 0.023013017 & negative \\
\hline CHST2 & IGFBP2 & 0.872735878 & 0.000211527 & positive \\
\hline IFITM1 & IGFBP2 & 0.757047546 & 0.00435922 & positive \\
\hline INHBA & IGFBP2 & -0.749747119 & 0.004987399 & negative \\
\hline LPAR1 & IGFBP2 & 0.593798189 & 0.041783501 & positive \\
\hline MXD1 & IGFBP2 & -0.794320094 & 0.002028742 & negative \\
\hline PTGER4 & IGFBP2 & 0.788471206 & 0.002309513 & positive \\
\hline RIPK2 & IGFBP2 & -0.719995108 & 0.008277386 & negative \\
\hline SLC4A4 & IGFBP2 & -0.745148832 & 0.005416701 & negative \\
\hline TLR2 & IGFBP2 & 0.604987195 & 0.037142038 & positive \\
\hline TNFRSF1B & IGFBP2 & 0.728583385 & 0.007197474 & positive \\
\hline BDKRB1 & SAMHD1 & -0.722948357 & 0.007893244 & negative \\
\hline CD82 & SAMHD1 & 0.752408175 & 0.004750999 & positive \\
\hline
\end{tabular}




\begin{tabular}{|lllll|}
\hline CDKN1A & SAMHD1 & -0.604274036 & 0.037426353 & negative \\
\hline IL1R1 & SAMHD1 & 0.639525823 & 0.02513441 & positive \\
\hline IL6 & SAMHD1 & 0.587393356 & 0.044618005 & positive \\
\hline PTGER2 & SAMHD1 & -0.579363545 & 0.048360329 & negative \\
\hline RIPK2 & SAMHD1 & 0.586135805 & 0.045190099 & positive \\
\hline SCN1B & SAMHD1 & -0.655653831 & 0.020621179 & negative \\
\hline SLC4A4 & SAMHD1 & -0.636717191 & 0.025987976 & negative \\
\hline C5AR1 & SAMHD1 & -0.582409884 & 0.046915516 & negative \\
\hline CD82 & TMTC1 & 0.714209971 & 0.009070195 & positive \\
\hline CXCR6 & TMTC1 & 0.721169657 & 0.00812297 & positive \\
\hline LPAR1 & TMTC1 & 0.833635546 & 0.000753658 & positive \\
\hline MXD1 & TMTC1 & 0.608111384 & 0.035914653 & positive \\
\hline PTGER2 & TMTC1 & -0.63942472 & 0.025164779 & negative \\
\hline TNFAIP6 & TMTC1 & 0.822676097 & 0.001016776 & positive \\
\hline TNFSF9 & TMTC1 & -0.582544685 & 0.046852295 & negative \\
\hline CXCR6 & TMTC1 & -0.677741891 & 0.015438898 & negative \\
\hline FZD5 & PTGER2 & 0.656214723 & 0.020475727 & positive \\
\hline IL6 & PTGER2 & 0.69943985 & 0.011350871 & positive \\
\hline PTGER2 & PTGER2 & 0.603262432 & 0.037832306 & positive \\
\hline RAF1 & PTGER2 & -0.718552304 & 0.00847006 & negative \\
\hline TNFAIP6 & -0.586483484 & 0.045031415 & negative \\
\hline TNFSF9 & -0.63902551 & 0.02528495 & negative \\
\hline
\end{tabular}

\section{Figures}




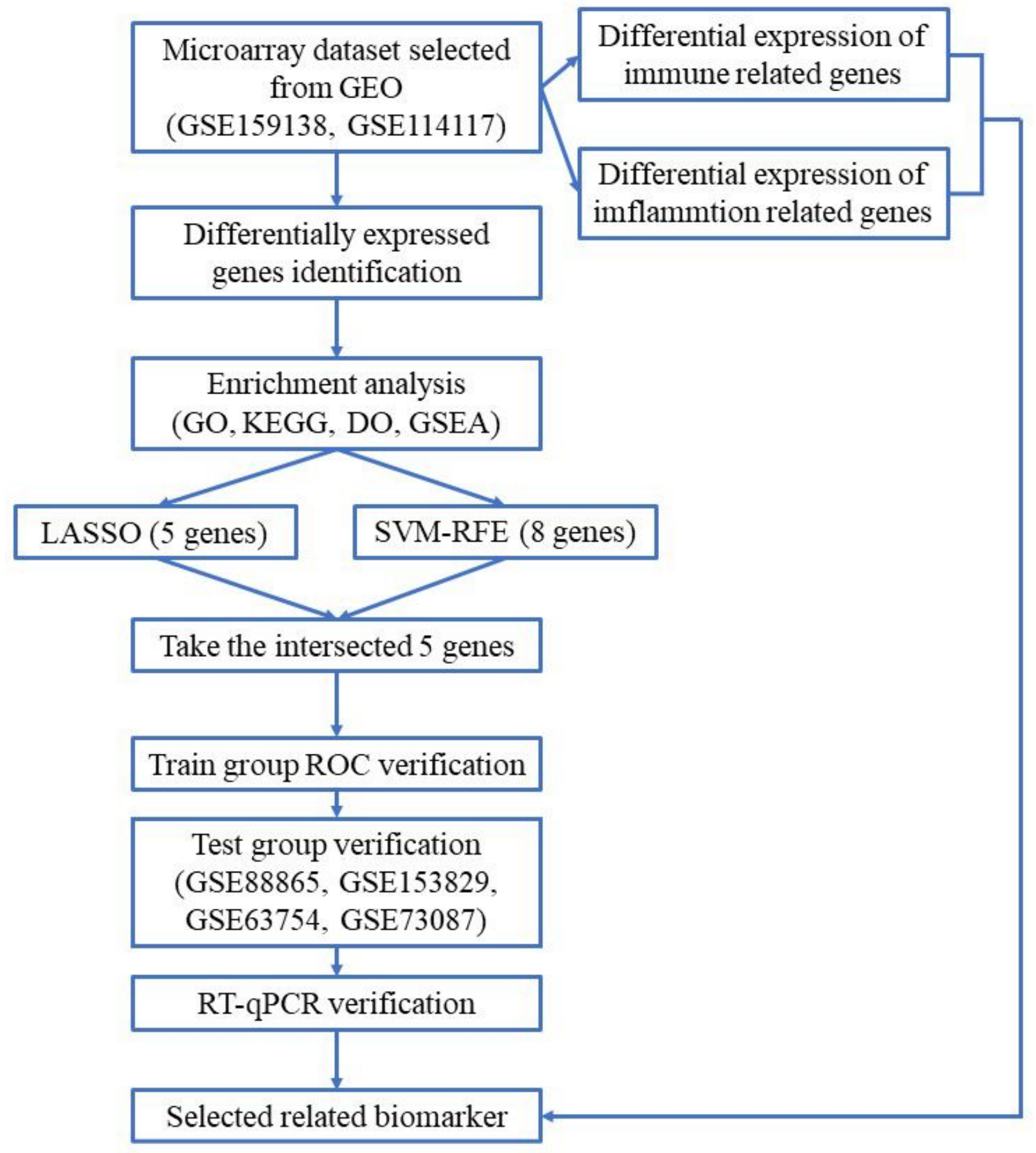

Figure 1 | Workflow chart of data generation and analysis.

Figure 1

See image above for figure legend. 
A

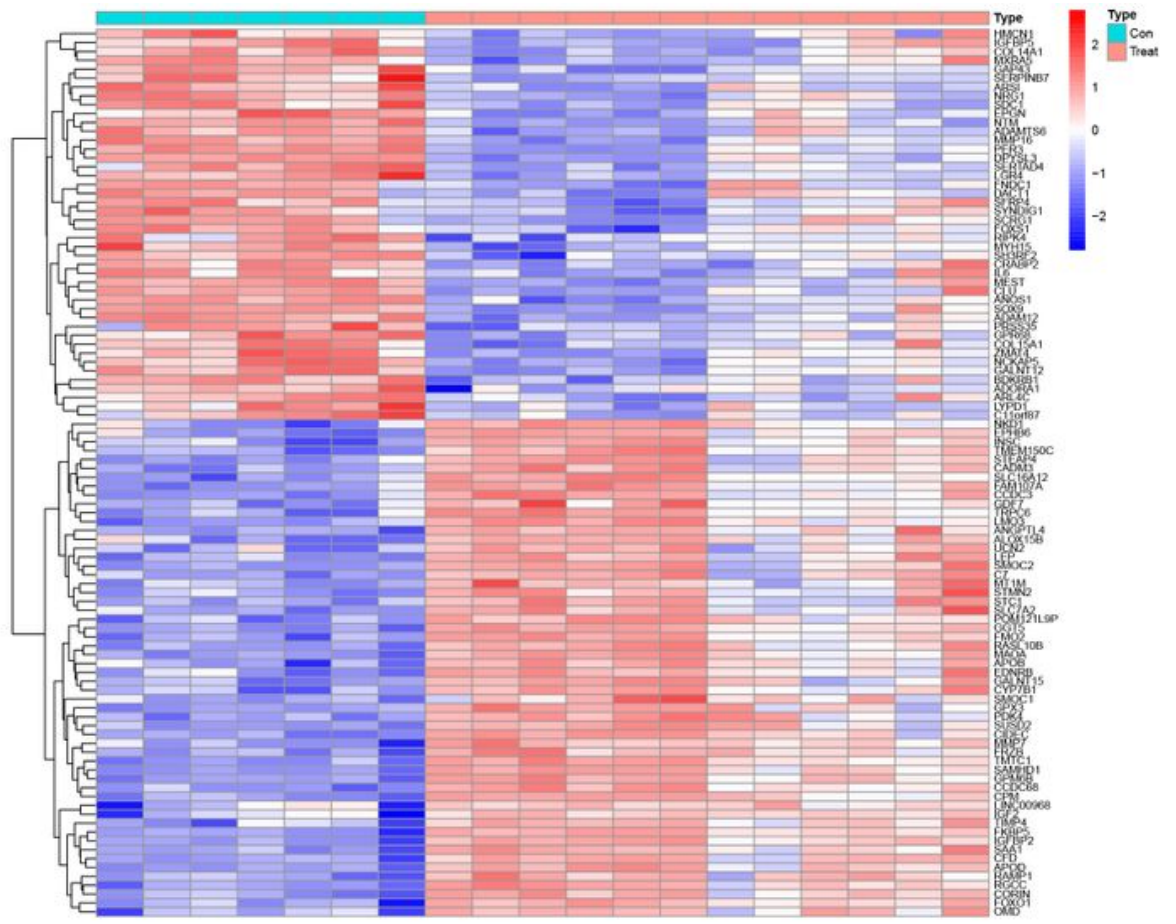

B

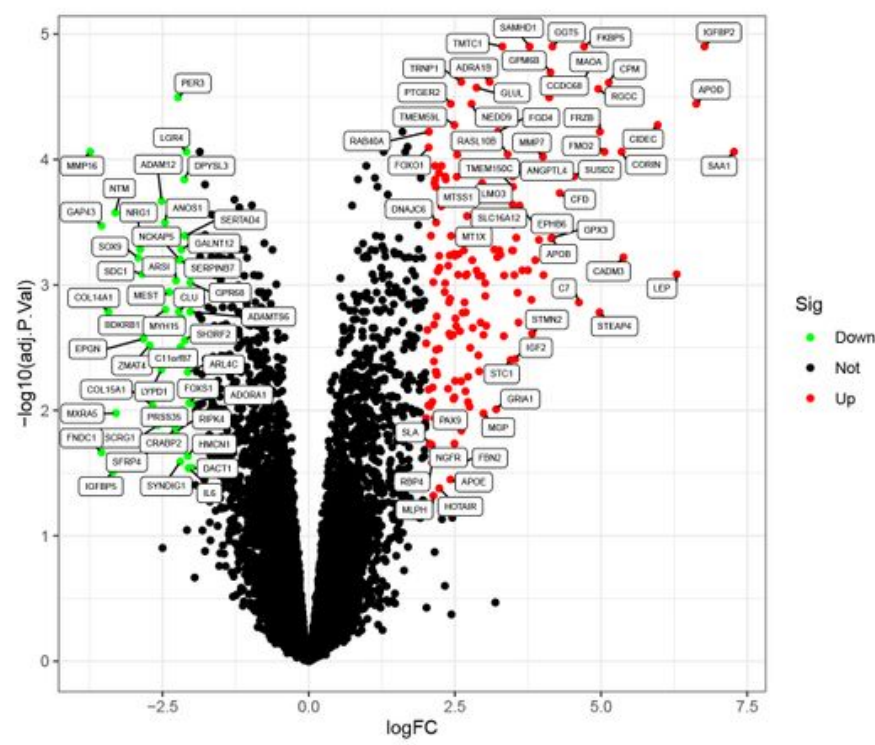

Figure 2 | The expression characteristics of genes granulosa cells in osteogenic mesenchymal stem cells (treat group) and uninduced mesenchymal stem cells (control group). (A) Heatmap presented the overall expression FDR $<0.05$ in treat and control group. (B) Volcano plot visualized the upregulated (red) and downregulated (blue) genes in treat and control group.

\section{Figure 2}

See image above for figure legend. 


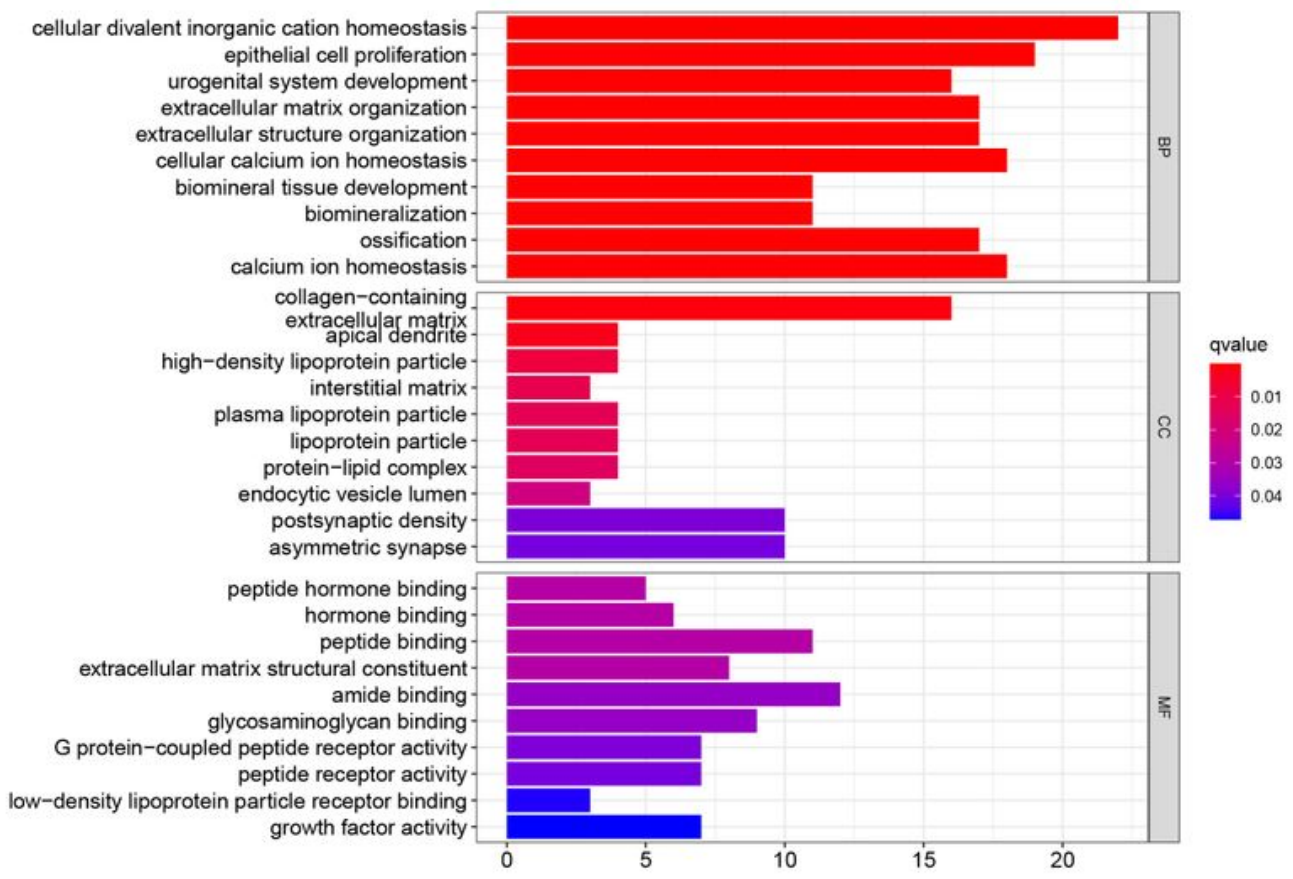

B

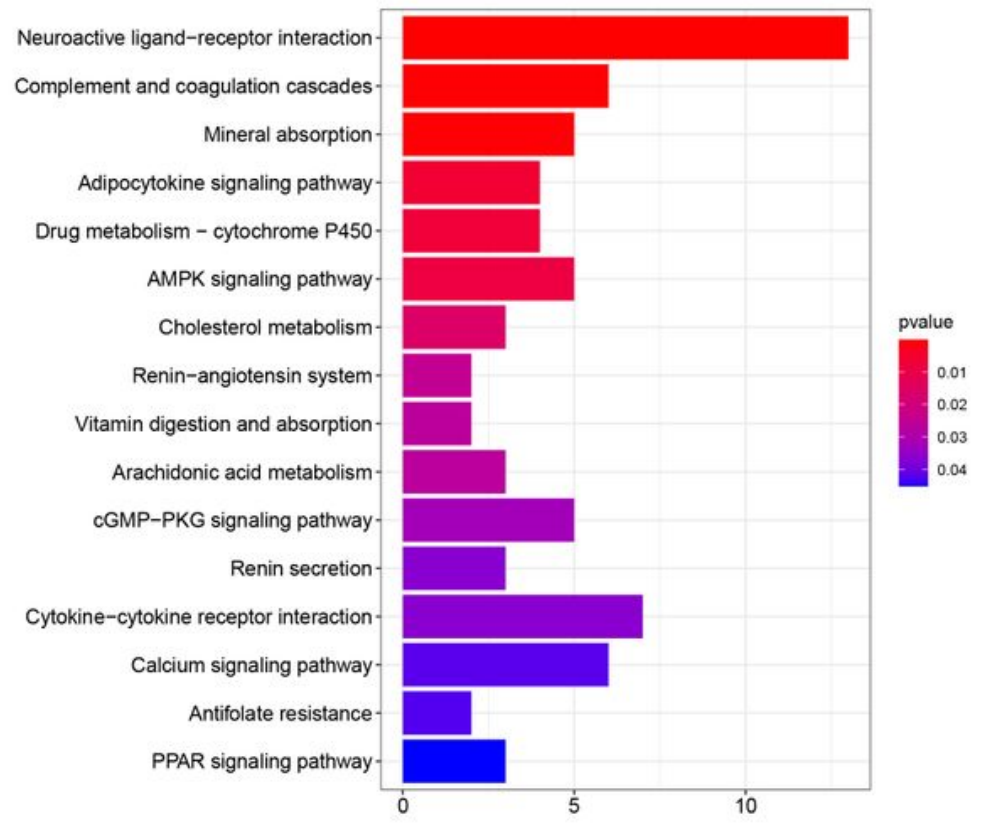

Figure 3 | (A) Gene Ontology (GO) analyses were conducted to predict the potential function of the differentially expressing genes between the treat and the control group. cellular component (CC), molecular function (MF), biological process (BP), respectively. (B) Kyoto Encyclopedia of Genes and Genomes (KEGG) potential pathways regarding the differentially genes between the treat and the control group.

\section{Figure 3}

\section{See image above for figure legend.}


A

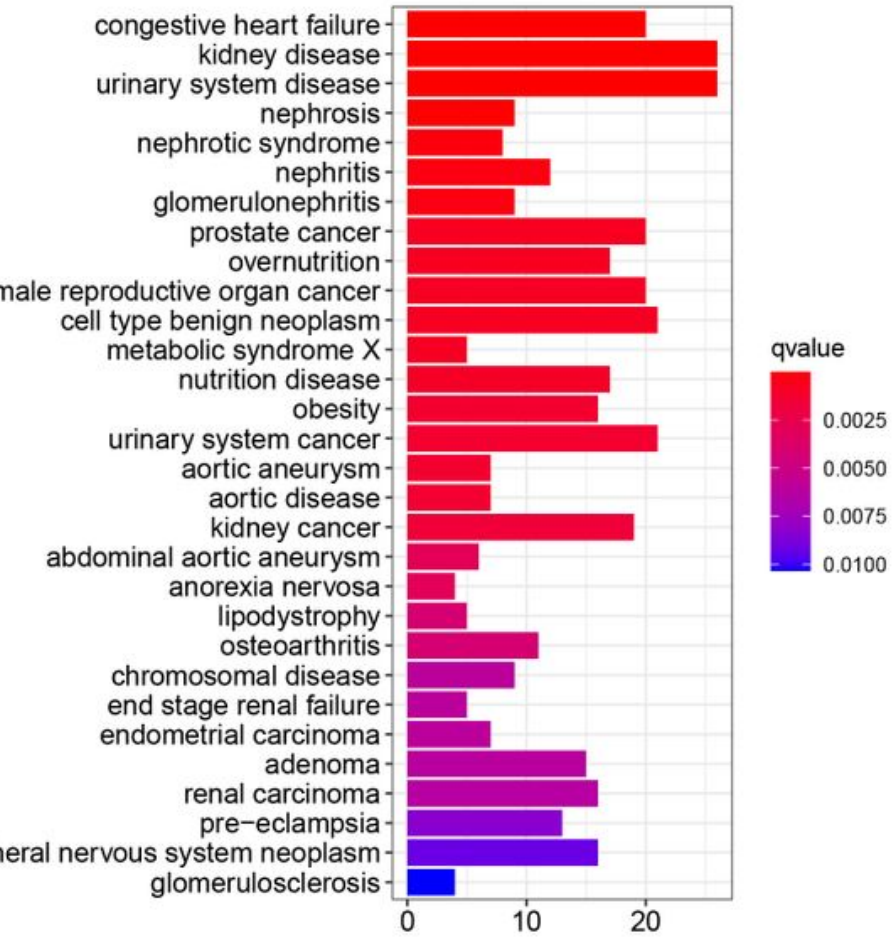

B

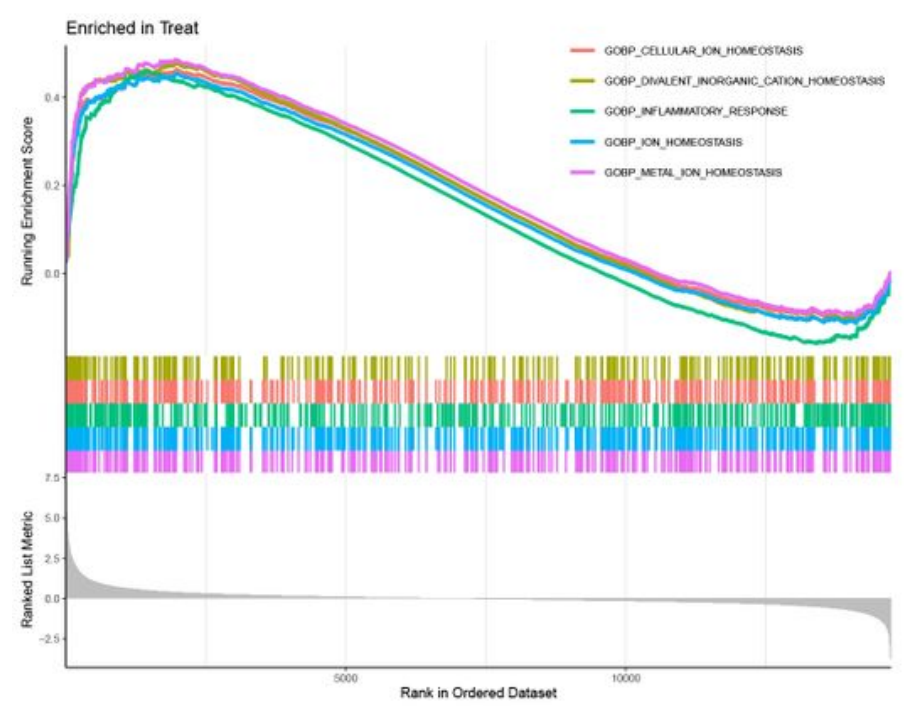

C

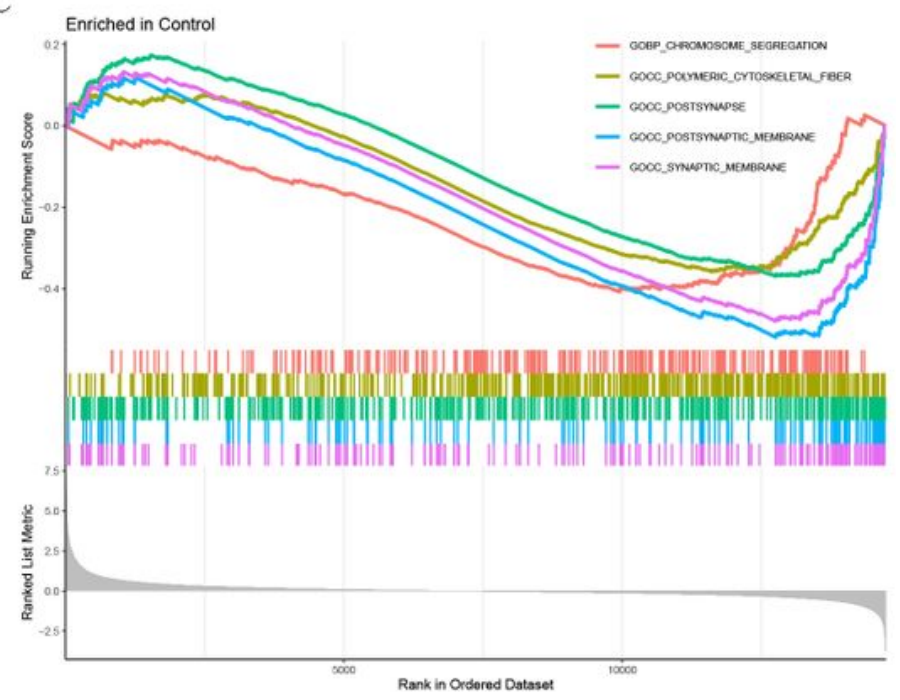

Figure $4 \mid$ (A) Disease ontology (DO) analyses were conducted to predict the potential related diseases of the differentially expressing genes between the treat and the control group. (B-C) GSEA showed that the top 5 signal pathways were most related to treat(B) and control group(C).

\section{Figure 4}

See image above for figure legend. 

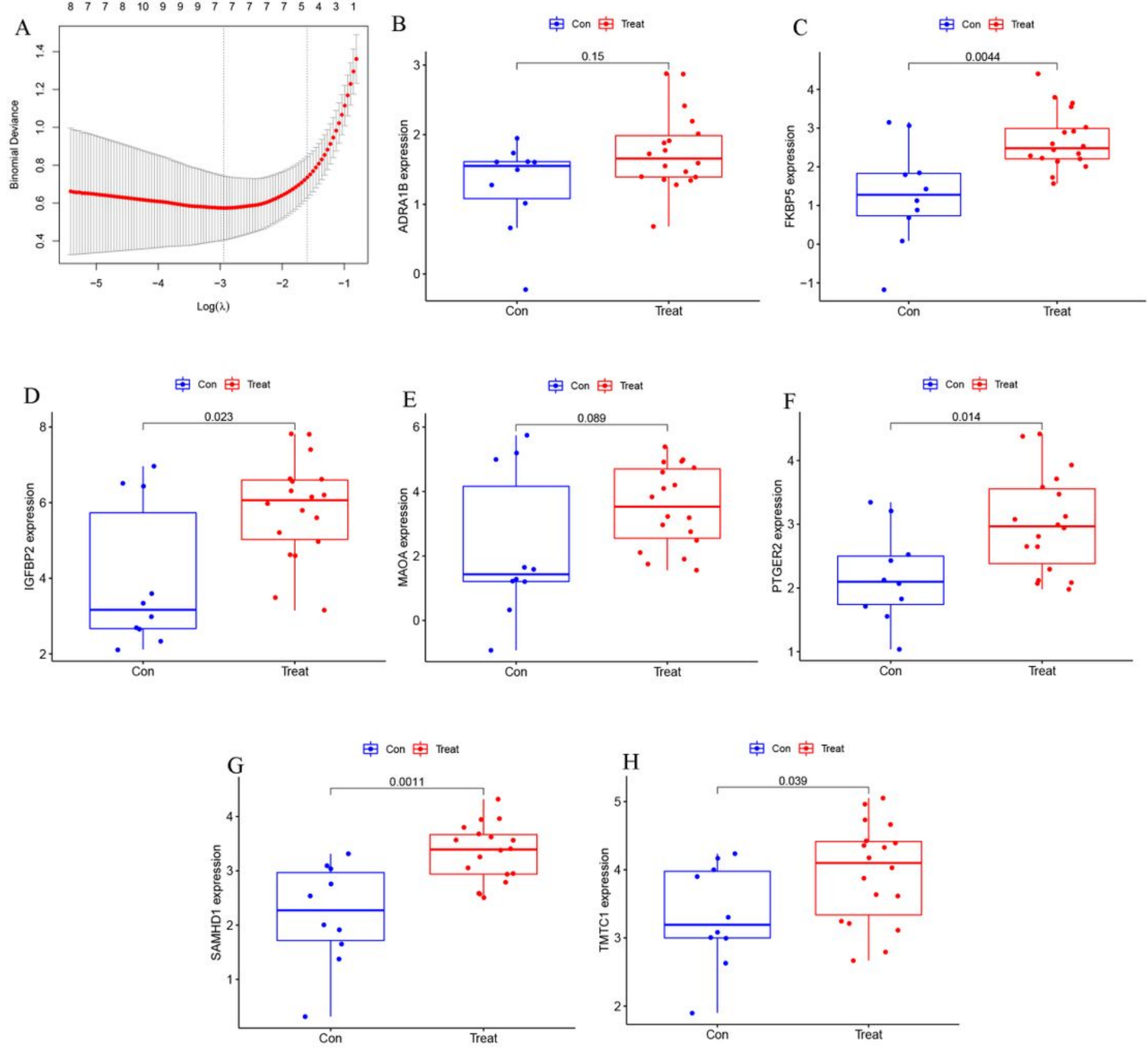

Figure $5 \mid$ (A) The least absolute shrinkage and selection operator (LASSO) logistic regression algorithm is used to retain the most predictive features. (B-H) The differential expression of ADRA1B (B), FKBP5(C), IGFBP2(D), MAOA(E), PTGER2 (F), SAMHD1(G) and TMTC1(H) in test group.

\section{Figure 5}

See image above for figure legend. 

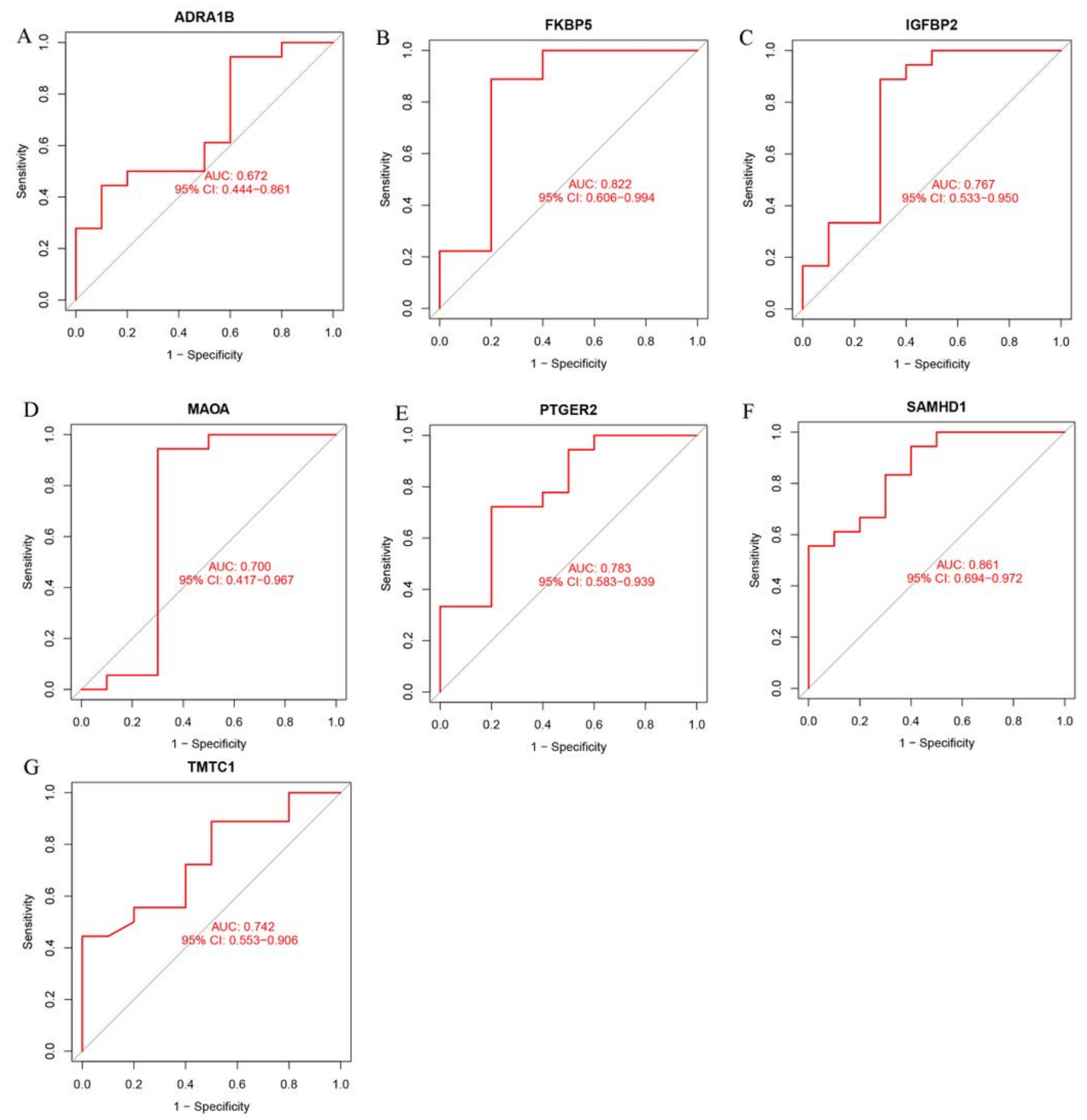

Figure $6 \mid$ (A-G) Receiver operating characteristic (ROC) curves of ADRA1B(A), FKBP5(B), IGFBP2(C), MAOA(D), PTGER2(E), SAMHD1(F) and TMTC1(G) in test group.

\section{Figure 6}

See image above for figure legend. 
A

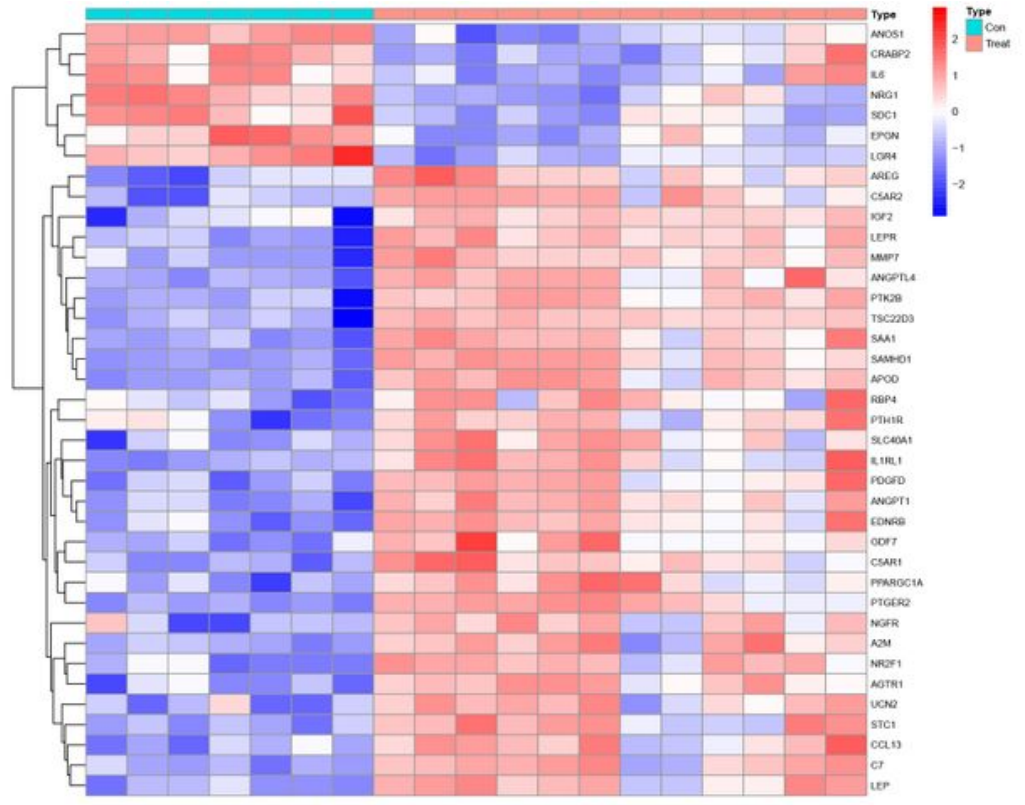

B

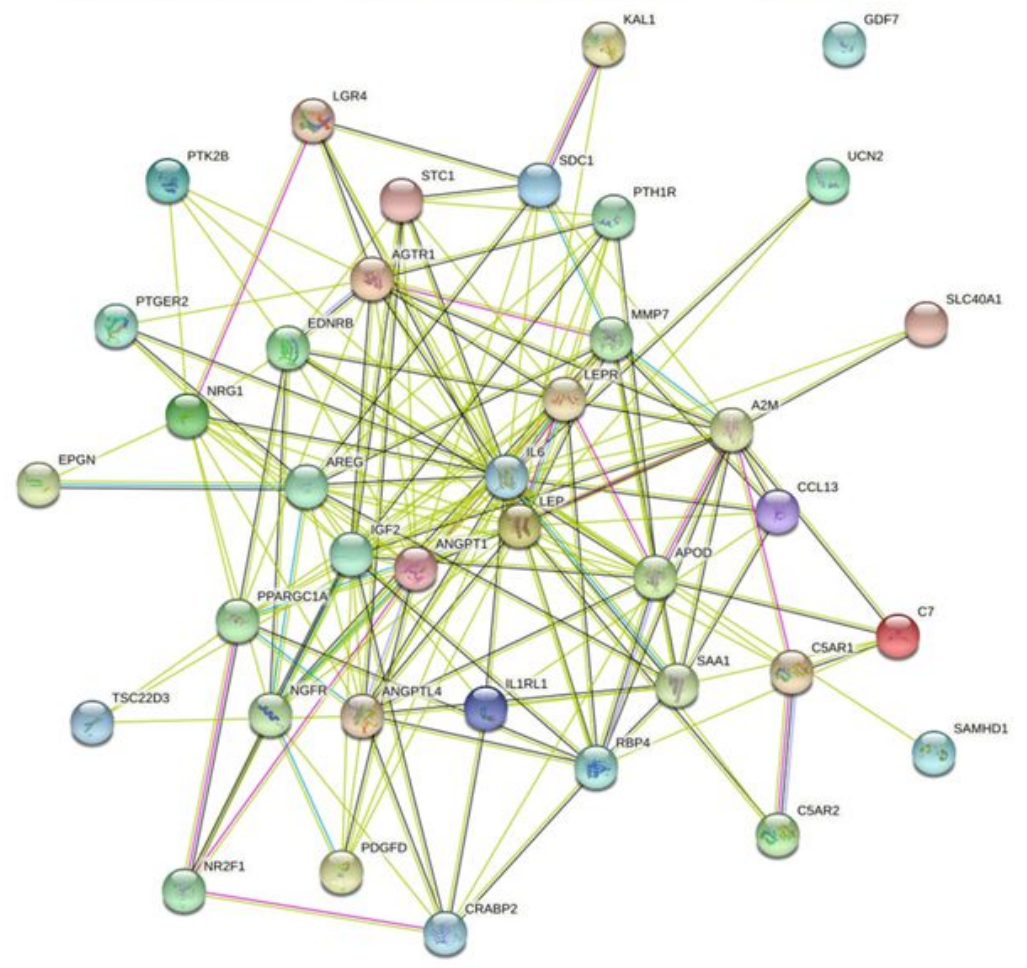

Figure $7 \mid$ The expression characteristics of immune related regulators. (A) Heatmaps presented the expression of inflammation related regulators in treat and control group. (B) The STRING Protein-Protein Interaction Networks of immune related regulators.

\section{Figure 7}

See image above for figure legend. 


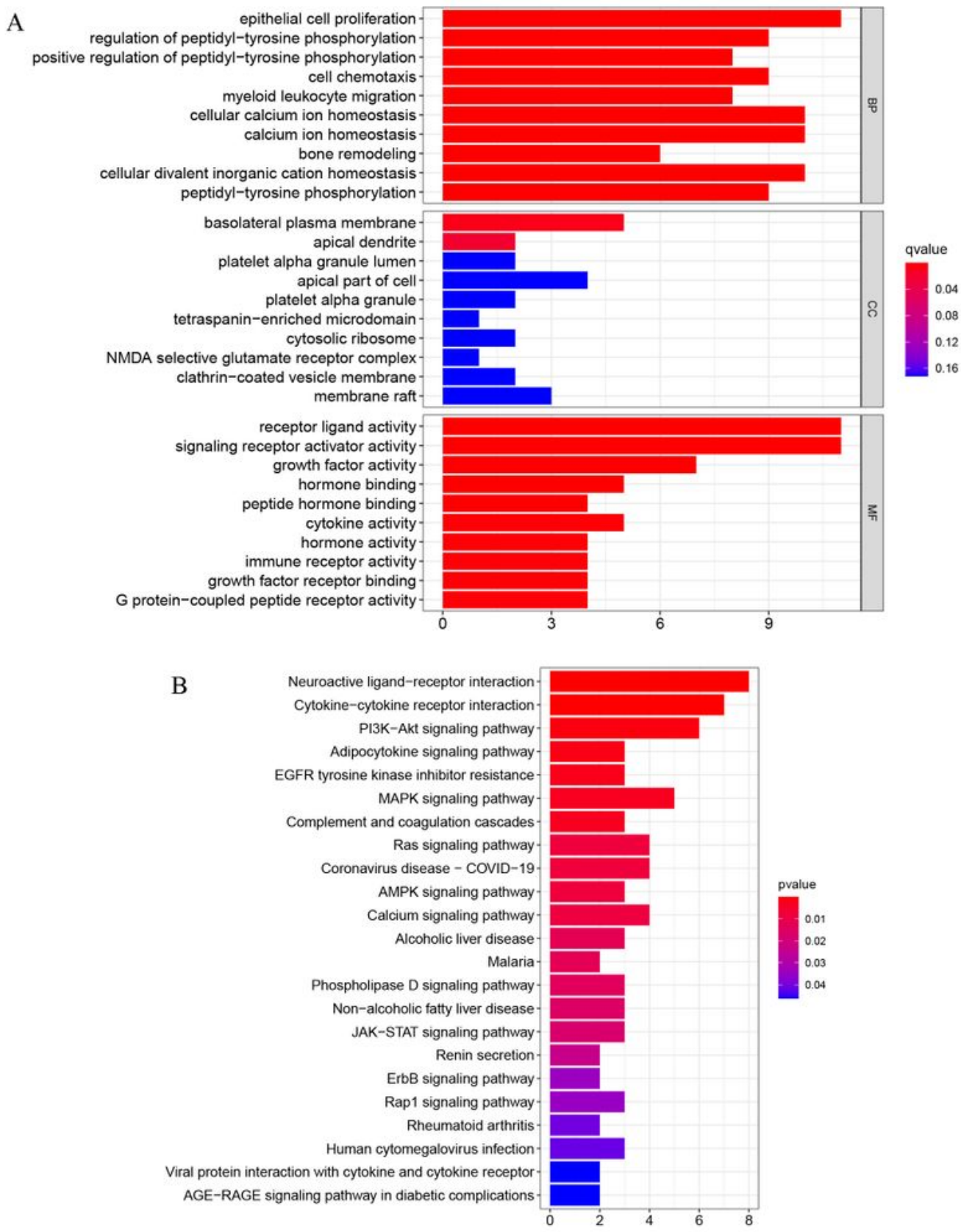

Figure $8 \mid$ (A) Gene Ontology (GO) analyses were conducted to predict the potential function of the differentially expressing immune related genes between the treat and the control group. cellular component (CC), molecular function (MF), biological process (BP), respectively. (B) Kyoto Encyclopedia of Genes and Genomes (KEGG) potential pathways regarding the differentially immune related genes between the treat and the control group.

\section{Figure 8}

See image above for figure legend. 
A
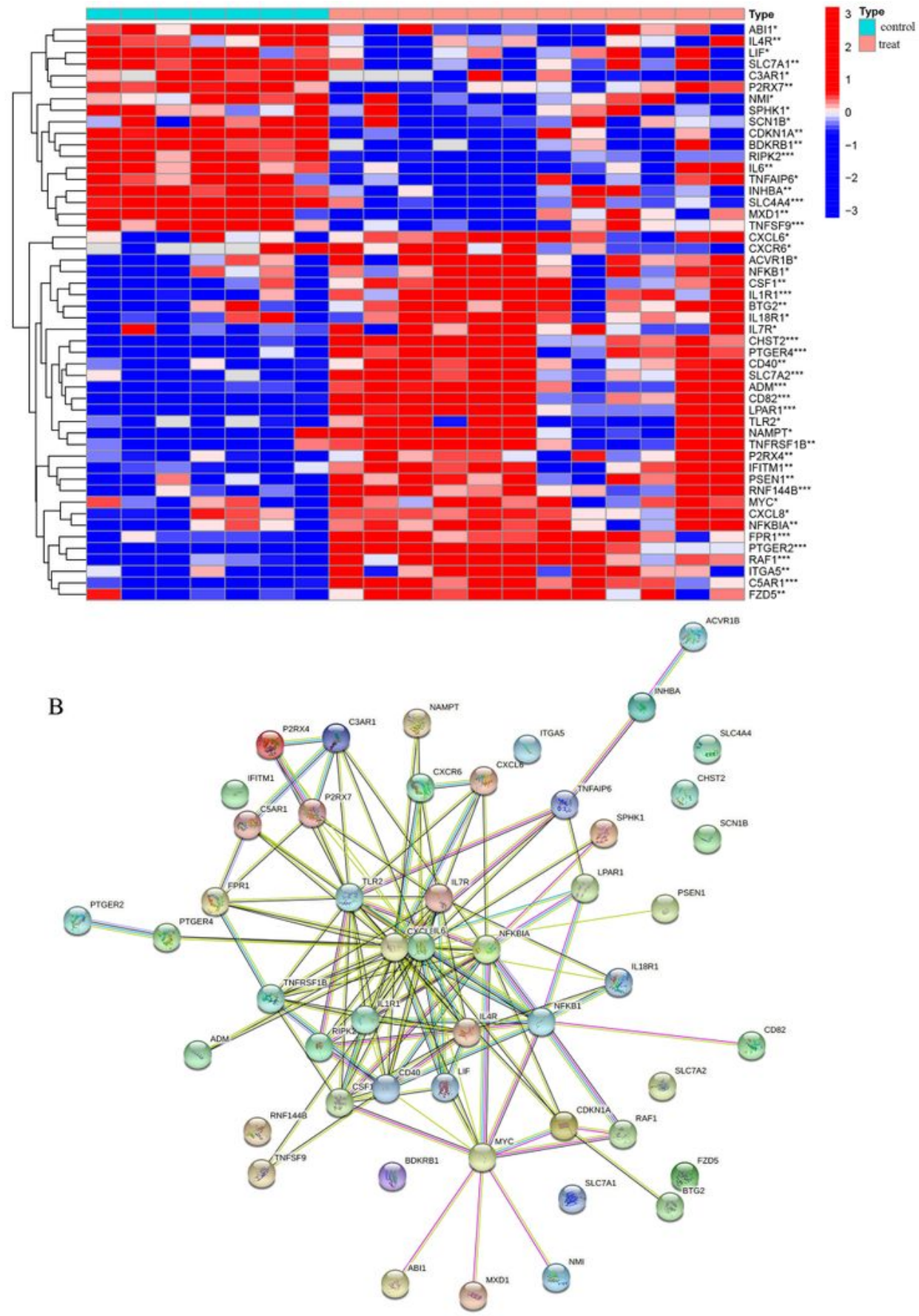

Figure $9 \mid$ The expression characteristics of inflammation related regulators. (A) Heatmaps presented the expression of inflammation related regulators in treat and control group. $\mathrm{P}<0.05$ (“*”), $\mathrm{P}<0.01$ (“**”) and $\mathrm{P}<0.01$ (“***”)

(B) The STRING Protein-Protein Interaction Networks of inflammation related regulators.

\section{Figure 9}

See image above for figure legend. 
A

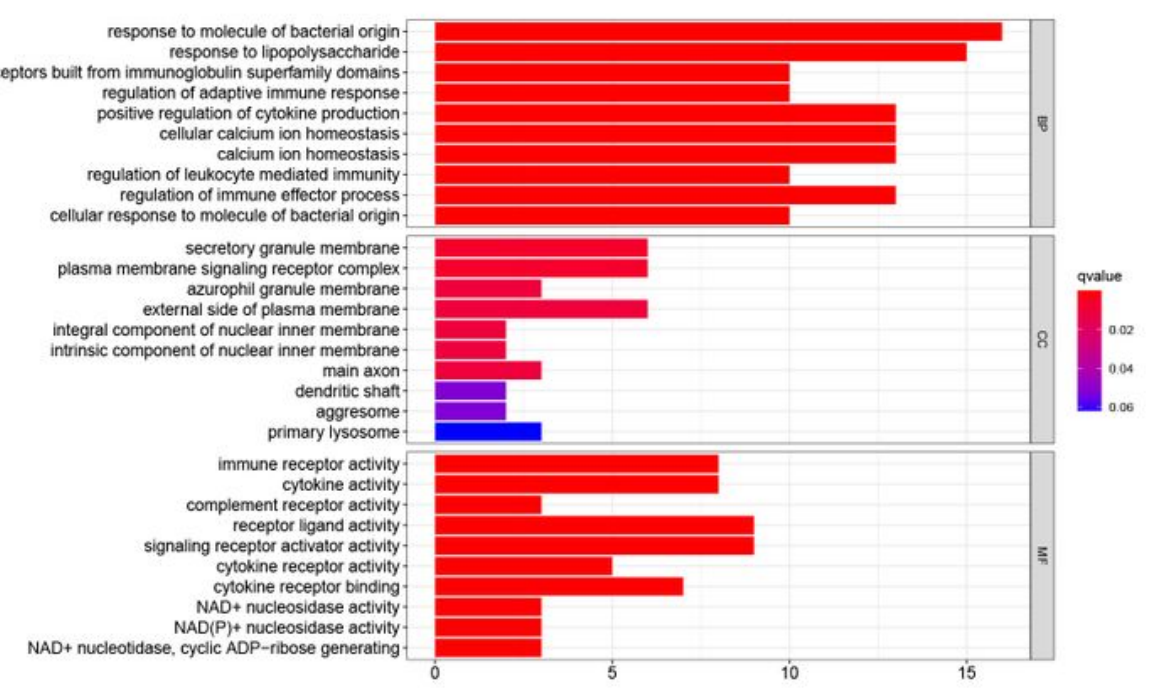

B

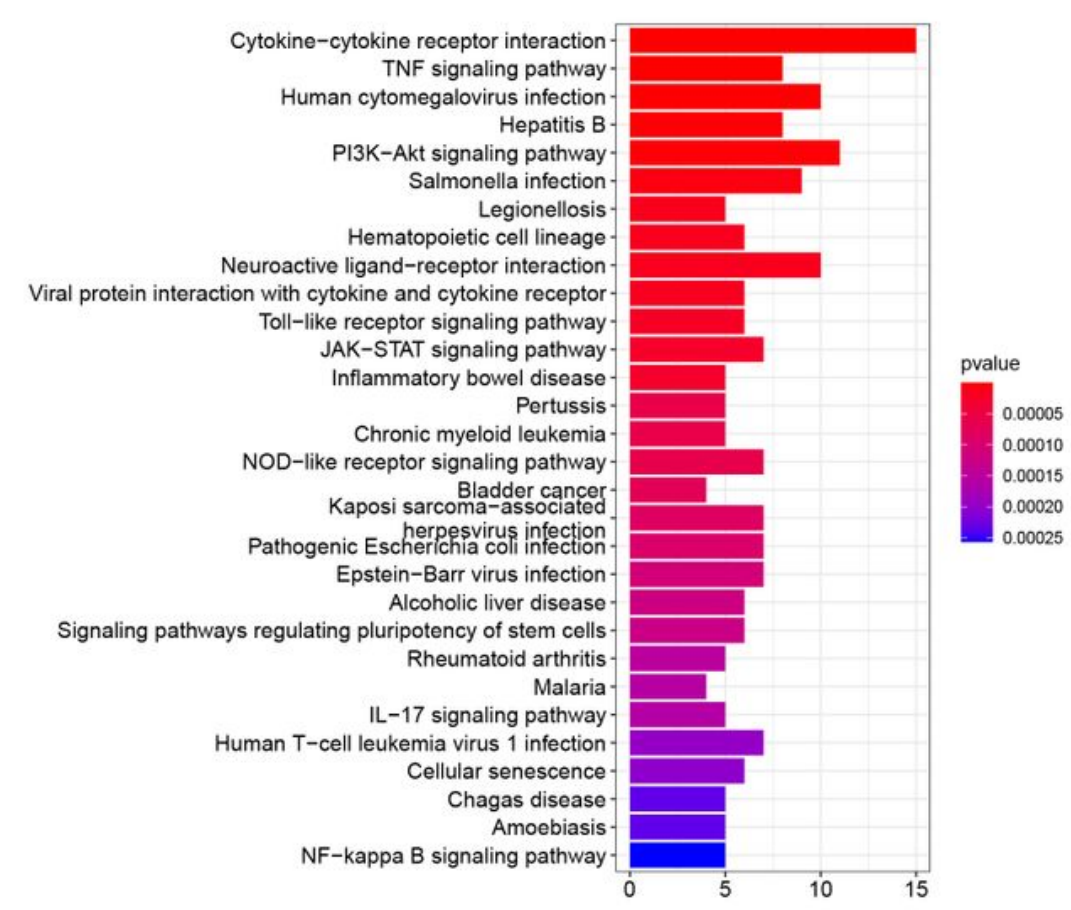

Figure $10 \mid$ (A) Gene Ontology (GO) analyses were conducted to predict the potential function of the differentially expressing inflammation related genes between the treat and the control group. cellular component (CC), molecular function (MF), biological process (BP), respectively. (B) Kyoto Encyclopedia of Genes and Genomes (KEGG) potential pathways regarding the differentially inflammation related genes between the treat and the control group.

\section{Figure 10}

See image above for figure legend. 
- biomarker - immnue related gene

A

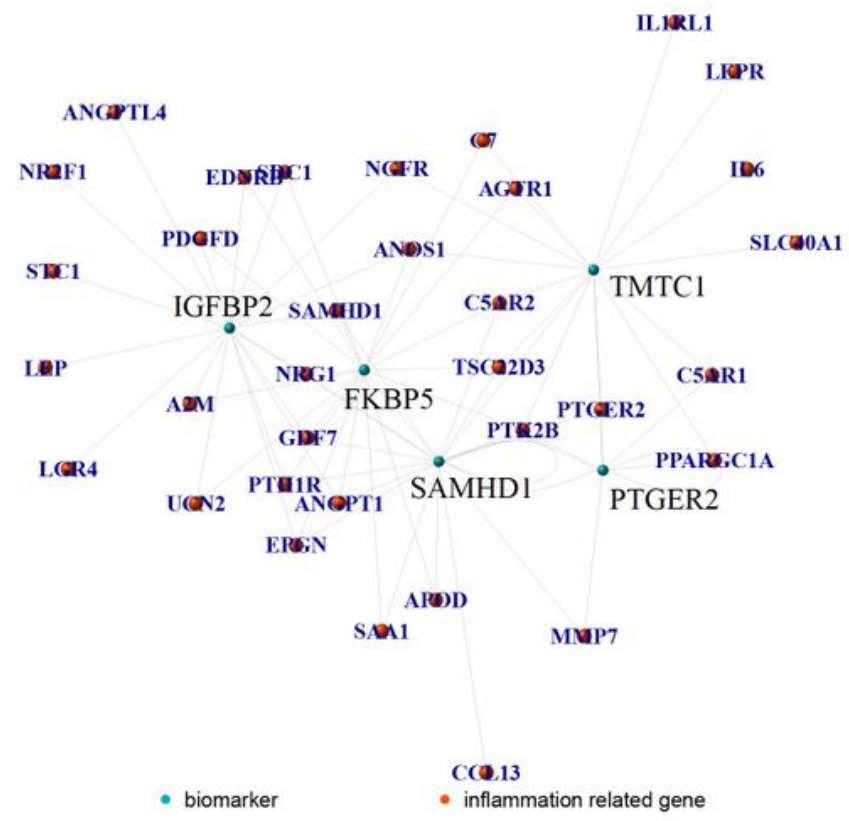

B

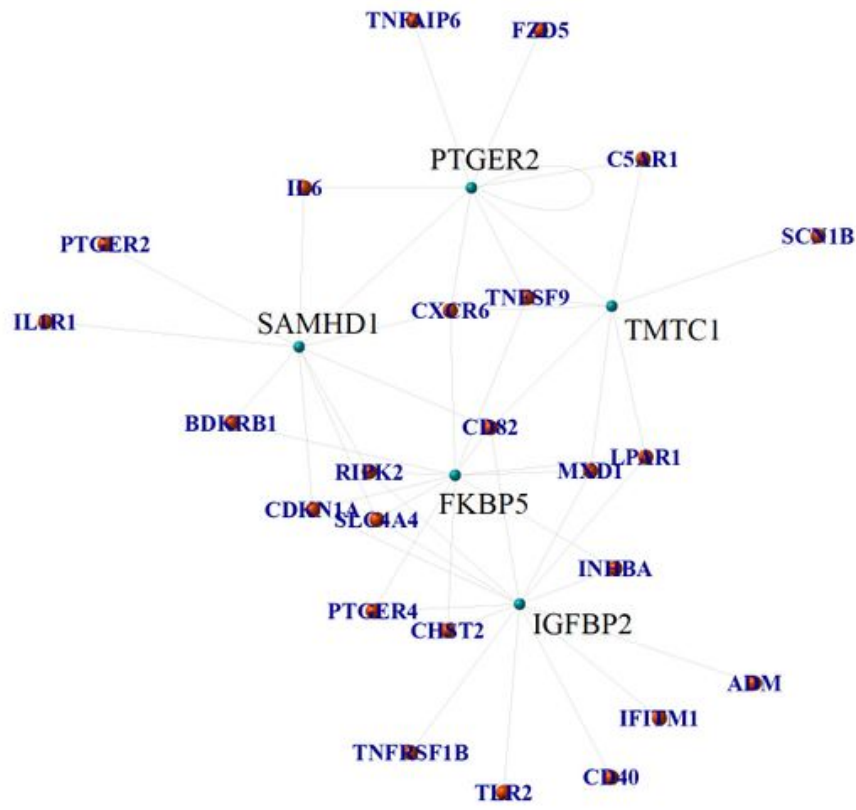

Figure $11 \mid$ (A) Relationship between differentially expressed immune related genes and selected biomarkers

(B) Relationship between differentially expressed inflammation related genes and selected biomarkers.

\section{Figure 11}

See image above for figure legend. 